\title{
Systemic arterial gas embolism (SAGE) as a complication of bronchoscopic lung biopsy: a case report and systematic literature review
}

\author{
Kai E. Swenson ${ }^{1,2}$, Brian D. Shaller ${ }^{3}$, Kevin Duong ${ }^{3}$, Harmeet Bedi $^{3}$ \\ ${ }^{1}$ Division of Pulmonary and Critical Care Medicine, Massachusetts General Hospital, Boston, MA, USA; ${ }^{2}$ Division of Pulmonary, Critical Care and \\ Sleep Medicine, Beth Israel Deaconess Medical Center, Boston, MA, USA; ${ }^{3}$ Division of Pulmonary, Allergy, and Critical Care Medicine, Stanford \\ University School of Medicine, Stanford, CA, USA \\ Contributions: (I) Conception and design: All authors; (II) Administrative support: None; (III) Provision of study materials or patients: None; (IV) \\ Collection and assembly of data: KE Swenson; (V) Data analysis and interpretation: All authors; (VI) Manuscript writing: All authors; (VII) Final \\ approval of manuscript: All authors. \\ Correspondence to: Kai E. Swenson. 55 Fruit St., BUL 148, Boston, MA 02114, USA. Email: keswenson@mgh.harvard.edu.
}

\begin{abstract}
Background: Systemic arterial gas embolism (SAGE) is a rare yet serious and underrecognized complication of bronchoscopic procedures. A recent case of presumed SAGE after transbronchial needle aspiration prompted a systematic literature review of SAGE after biopsy procedures during flexible bronchoscopy.

Methods: We performed a systematic database search for case reports and case series pertaining to SAGE after bronchoscopic lung biopsy; reports or series involving only bronchoscopic laser therapy or argon plasma coagulation (APC) were excluded. Patient data were extracted directly from published reports.

Results: A total of 29 unique patient reports were assessed for patient demographics, specifics of the procedure, clinical manifestations, diagnostic findings, and clinical outcomes. Cases of SAGE occurred after multiple types of bronchoscopic biopsy and under both positive and negative pressure ventilation. The most common clinical findings were neurologic, followed by cardiac manifestations; temporal patterns included acute onset of cardiac or neurologic emergencies immediately after biopsy, or delayed awakening post-procedure. There was a high mortality rate among cases $(28 \%)$, with residual neurologic deficits also common (24\%).

Discussion: SAGE is an underrecognized but severe adverse effect of bronchoscopic lung biopsy, which often presents with acute coronary or cerebral ischemia or delayed awakening from sedation. It is important for all physicians who perform bronchoscopic biopsies to be aware of the clinical manifestations and therapeutic management of SAGE in order to mitigate morbidity and mortality among patients undergoing these procedures.
\end{abstract}

Keywords: Bronchoscopy; transbronchial biopsy; gas embolism; air embolism

Submitted Apr 23, 2021. Accepted for publication Sep 24, 2021.

doi: $10.21037 /$ jtd-21-717

View this article at: https://dx.doi.org/10.21037/jtd-21-717

\section{Introduction}

Flexible bronchoscopy is a common procedure which is essential for the diagnosis of many pulmonary conditions, with very low rates of significant complication (1). However, as training in bronchoscopy grows, an increasing number of providers are routinely performing a variety of endobronchial and transbronchial biopsies. It is therefore incumbent on these practitioners to be aware of rare complications of such procedures.

Systemic arterial gas embolism (SAGE), also known 

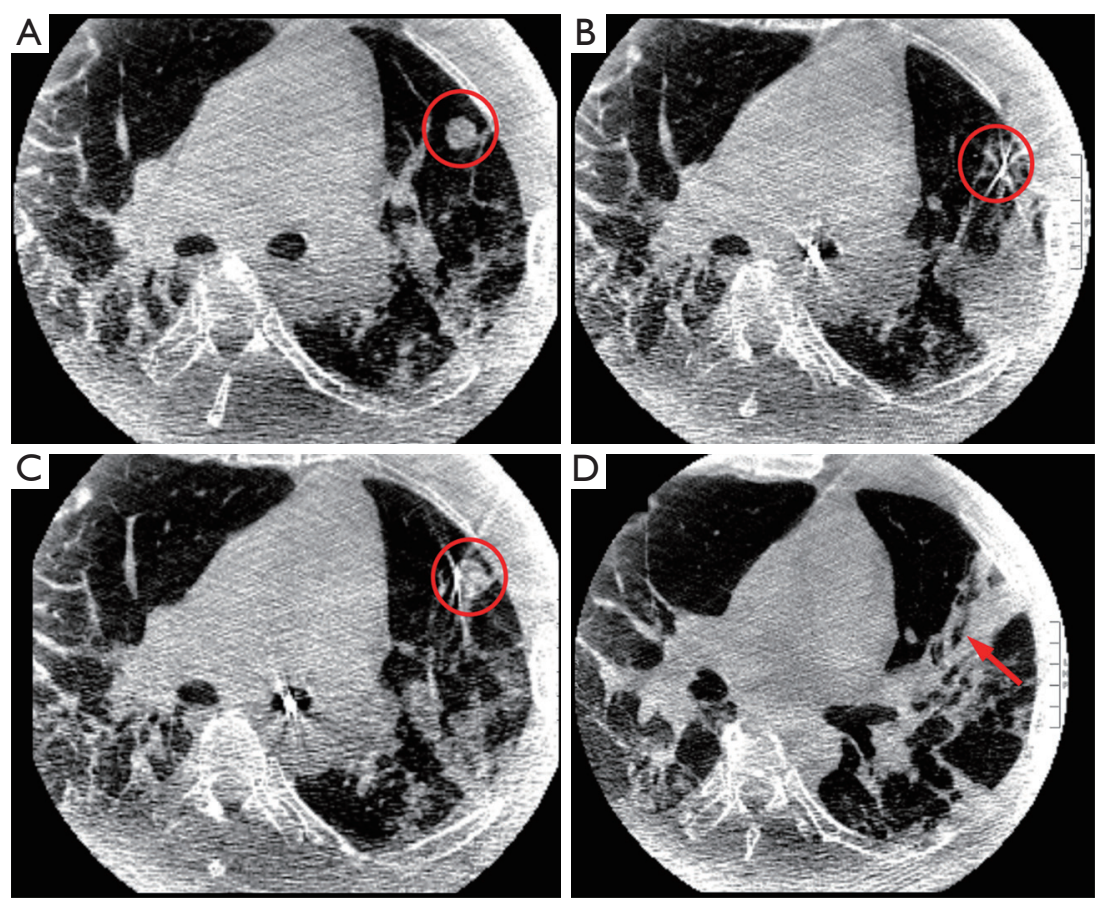

Figure 1 Cone-beam computed tomography images obtained during bronchoscopic procedure. (A) Initial image demonstrated the lingular nodule of interest (red circle). (B,C) Images obtained during initial biopsy, demonstrating confirmation of biopsy tool-in-lesion (red circles). (D) Image obtained after biopsies, demonstrating progressive lingular atelectasis (red arrow) which prompted recruitment maneuvers.

as systemic arterial air embolism (SAAE), may occur during bronchoscopy when gas bubbles enter the pulmonary venous circulation and embolize to systemic arterial vascular beds. Other emergent complications of bronchoscopy-such as severe airway hemorrhage or tension pneumothorax - are much more widely recognized than SAGE; as such, the incidence, clinical presentation, and management of SAGE remain underappreciated. Even among interventional pulmonologists, gas embolism has predominantly been considered a risk of local airway wall therapies, including argon plasma coagulation (APC) therapy of the airway wall as described by others $(2,3)$; however these reviews do not mention instances of SAGE occurring after bronchoscopic biopsy procedures. Herein we present a systematic review of the published case literature on SAGE after bronchoscopic biopsy procedures, followed by a discussion of the pathophysiology, risk factors, clinical manifestations, diagnosis, treatment, prognosis, and prevention of this condition. Our review begins with a recent case of presumed SAGE at our institution as a means of highlighting certain key aspects of the presentation, diagnosis and clinical management.

We present the following article in accordance with the
PRISMA reporting checklist (available at https://dx.doi. org/10.21037/jtd-21-717).

\section{Case presentation}

A 48-year-old former smoker with obesity underwent flexible bronchoscopy with cone beam CT (CBCT)-guided navigational biopsy of an incidentally-discovered $1.4-\mathrm{cm}$ lingular nodule. The procedure was performed in the supine position under general anesthesia. Initial biopsies of the lingular nodule were performed after tool-in-lesion confirmation by CBCT; however after the third biopsy, diffuse atelectasis had progressed significantly (Figure 1), necessitating the implementation of recruitment maneuvers via adjustable pressure limitation (APL) at $30-40 \mathrm{cmH}_{2} \mathrm{O}$. Approximately 15 seconds after the second recruitment maneuver was initiated, the patient became hypotensive and bradycardic, with complete heart block and a wide QRS complex on telemetry. Airway pressure was immediately released, and intravenous glycopyrrolate and epinephrine were administered. Hypotension quickly resolved, and the ventricular complex narrowed with heart rate improvement; however, complete heart block persisted and new large ST 

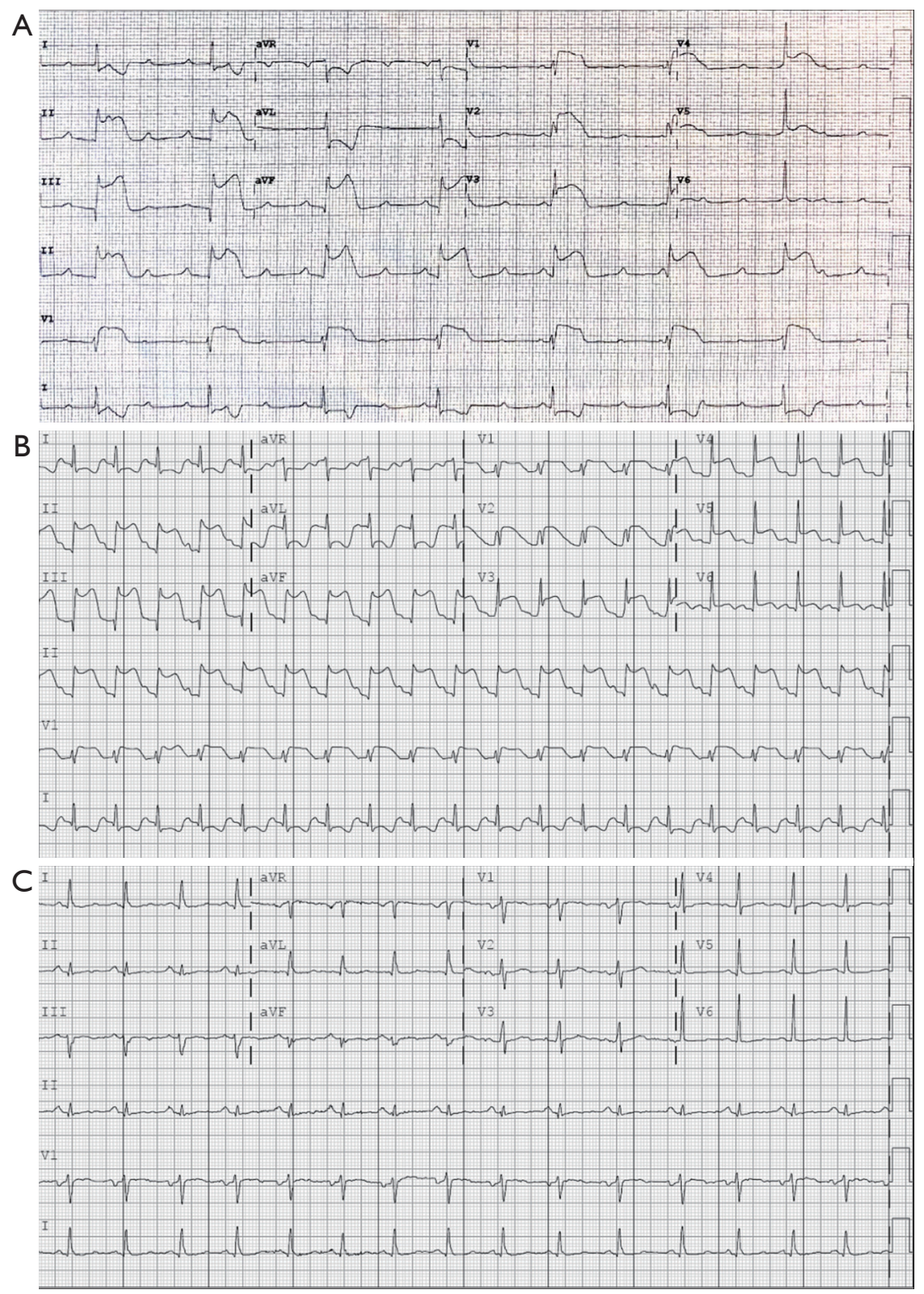

Figure 2 EKG during and after procedure. (A) During intraprocedural hypotension, EKG revealed inferior and anterior ST elevations (greatest in V1-V2), with reciprocal depression in lateral leads, as well as complete heart block, suggestive of proximal RCA involvement. (B) A repeat EKG, within 15 minutes and after resolution of hypotension, demonstrated resolution of heart block but persistent inferior and anterior ST elevations. (C) EKG postcoronary catheterization demonstrated resolution of prior ST segment changes. EKG, electrocardiography; RCA, right coronary artery.

segment elevations in the inferior and anterior leads were demonstrated on 12-lead EKG (Figure 2A), which persisted after the patient spontaneously converted back to sinus rhythm (Figure 2B). Emergent coronary catheterization, completed within 15 minutes, demonstrated normal coronary arteries without evidence of thromboembolic disease, vasospasm or dissection; a clinical diagnosis of coronary gas embolism was thought likely.

The procedure was terminated and the patient extubated successfully; however, he was slow to awaken and became 

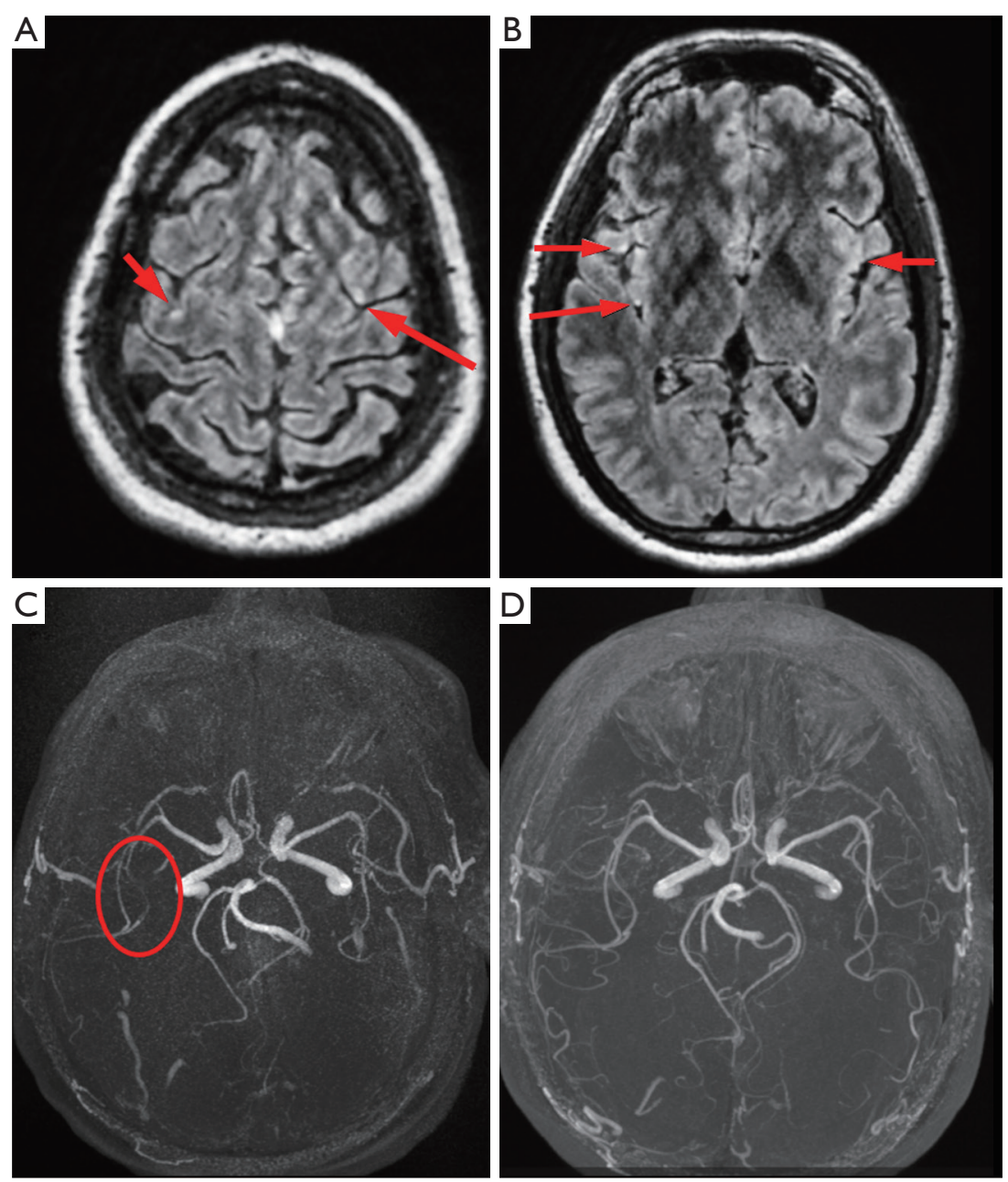

Figure 3 MRI of the brain performed at 6 hours post-procedure demonstrated multiple areas of gyral enhancement (red arrows, A,B), as well as poorly defined vascularity of bilateral MCA territories with possible occlusion of the right inferior M2 segment (red circle, C), which had resolved on follow-up MRI at 48 hours (D). MRI, magnetic resonance imaging; MCA, middle cerebral artery.

agitated without following commands. Neurologic exam demonstrated a right gaze preference, left facial droop, left arm monoplegia, and minimal left leg movement. An emergent head CT scan performed at 90 minutes revealed no intravascular air or large territory infarct; however, suboptimal opacification of the right M2 inferior segment was noted. Given the high clinical likelihood of gas embolism, thrombolytic therapy was deferred; the patient was placed on $100 \%$ supplemental oxygen via nonrebreather facemask and transferred to the intensive care unit. His neurologic status improved dramatically within the next 2 hours: he was alert and fully oriented, following commands, visually tracking across midline, and fully motor-intact with the exception of persistent left arm weakness. Transfer to a hyberbaric oxygen (HBOT)-capable facility was considered but deferred given the rapid and significant neurological improvement. Magnetic resonance imaging (MRI) of the brain performed at 6 hours revealed subtle areas of gyral enhancement in the middle cerebral artery (MCA) watershed territories of both hemispheres and redemonstrated possible occlusion of the right M2 inferior segment; these abnormalities fully resolved on follow-up MRI 48 hours later (Figure 3). A follow-up EKG demonstrated no residual ST segment changes (Figure 2C), and echocardiography revealed no wall motion abnormalities. The patient was discharged on hospital day 3 


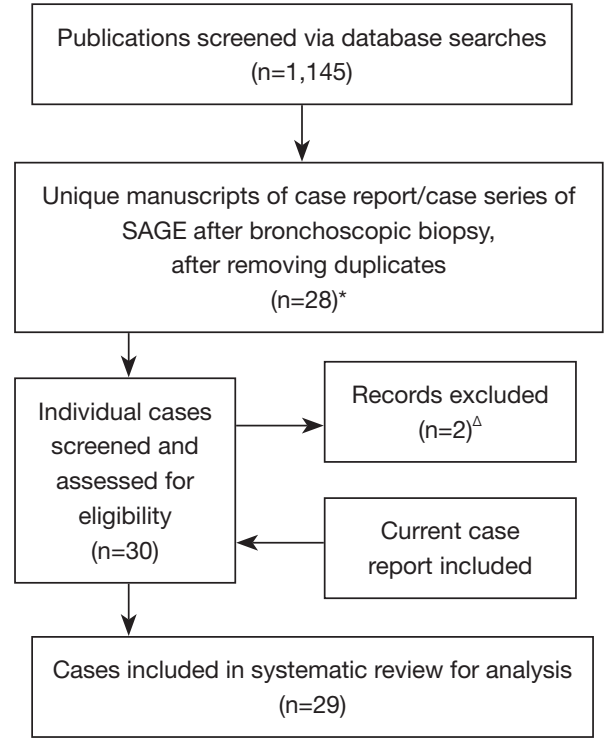

Figure 4 Modified PRISMA flow diagram for systematic review of case reports/series. *, includes 26 case reports and 2 case series, for 30 individual case reports; ${ }^{\Delta}$, explanation of excluded records is found in the methods section.

with no residual neurologic deficits.

\section{Methods}

We performed a systematic literature search in October 2020 for case reports and case series pertaining to SAGE in Medline, EMBASE, Web of Science, Cochrane Library, and SCOPUS. There was no funding source for this study. The search criteria utilized a combination of the

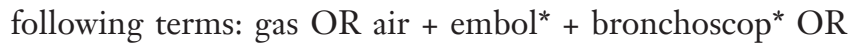
transbronch*. Studies were assessed for inclusion consistent with a PRISMA approach (Figure 4) (4). Case reports or series involving only bronchoscopic laser therapy or APC were excluded. After removing duplicates, 24 case reports (5-28) and 2 case series (each containing two individual case reports) $(29,30)$ of patients who presented with SAGE after bronchoscopic biopsy procedures were identified. The search revealed two reports of idiopathic complications during bronchoscopic procedures $(31,32)$ under clinical circumstances so remarkably similar to the presentation of SAGE that we included these in our review, for a total of 30 unique patient reports. The clinical circumstances from one case, presented at a national meeting, could not be obtained (25), and another case report was excluded due to the high likelihood that the cerebral gas embolism had occurred due to central line manipulation rather than bronchoscopy, leading to venous air embolism (26). In total, 29 individual reports (including the case reported above) were ultimately reviewed in detail and data extracted regarding patient demographics, procedural specifics, clinical manifestations, diagnostic findings, and clinical outcomes.

\section{Results}

The results of a systematic literature review of SAGE (Figure 4) during bronchoscopic biopsy procedures are shown in Tables 1,2. The average age of the patients was 65 years, with approximately 2:1 male predominance. Roughly one-half of patients had underlying lung disease, with obstructive lung disease being the most common. Most of the procedures were performed for suspected malignancy. Transbronchial forceps biopsies were performed in the majority $(55 \%)$ of cases, followed by transbronchial needle aspiration (38\%); however, some case reports of SAGE involved only endobronchial forceps biopsies or bronchial curettage, and one event likely occurred during oxygen insufflation of an obstructed bronchus prior to attempted biopsy (9). Although only reported in 19 of 29 cases, the majority of SAGE events occurred under moderate sedation $(63 \%)$, with a minority of cases involving topical sedation only $(21 \%)$ or general anesthesia $(16 \%)$, with a resulting majority of cases occurring while the patient was spontaneously breathing. Coughing at the time of biopsy was reported in $28 \%$ of cases, while moderate-to-severe bleeding (defined by a need for intervention, including cold saline or epinephrine instillation) was reported in $21 \%$.

Clinical manifestations involved both cerebral and cardiac abnormalities. Cerebral manifestations were present in $97 \%$ of cases, with the most common being focal deficits $(52 \%$, with a strong predilection for left sided involvement and hemiplegia), seizures (48\%) and acute intraprocedural unresponsiveness (45\%) - often within 30 seconds of the biopsy. Delayed post-procedural awakening with altered mental status was a presenting sign in $28 \%$ of cases. Cardiac complications were rarer (28\% of cases) and much less likely to be confirmed on imaging. In only 1 case (31) were there no reported cerebral manifestations alongside the stated cardiac complication, and in this case cerebral imaging was not performed. Among cases with cardiac manifestations, acute electrocardiographic abnormalities were common and included bradycardia, complete heart block, and ST elevations. Intraprocedural cardiac arrest occurred in $17 \%$ 
Table 1 Demographics and procedural specifics identified in case reports of systemic arterial gas embolism occurring during bronchoscopic biopsy procedures

\begin{tabular}{lc}
\hline Demographics & All patients $(\mathrm{n}=29)$ \\
\hline Age & $65 \pm 15$ \\
Gender & \\
Male & $17(65 \%)$ \\
Female & $9(35 \%)$ \\
Prior history of lung disease & \\
Obstructive & $8(28 \%)$ \\
Restrictive & $3(10 \%)$ \\
History of lung resection & $3(10 \%)$ \\
Procedure & \\
Types of biopsy performed & \\
Transbronchial forceps biopsy & \\
Transbronchial needle aspiration & $16(55 \%)$ \\
Endobronchial forceps biopsy & $11(38 \%)$ \\
Bronchial curettage & $2(7 \%)$ \\
No biopsy performed & \\
Type of sedation & \\
Topical analgesia only & $23(16 \%)$ \\
Moderate sedation & $2(7 \%)$ \\
General anesthesia & $1(3 \%)$ \\
\hline
\end{tabular}

Continuous measures reported as mean \pm standard deviation; categorical measures reported as number (percentage). *, reported in $26 / 29$ cases; ${ }^{\Delta}$, gas embolism occurred after oxygen insufflation prior to planned biopsy. ${ }^{\Phi}$, reported in 19/29 cases; ${ }^{\Psi}$, reported in 26/29 cases.

of cases. Confirmation of the presence of gas emboli was found in $79 \%$ of cases-predominantly on emergent CT head imaging.

Supplemental oxygen therapy was initiated in the vast majority of cases (and in all cases in which the responding clinician suspected SAGE); $55 \%$ received normobaric and $38 \%$ received hyperbaric oxygen therapy. Rapid clinical
Table 2 Clinical manifestations, diagnosis, treatment and outcomes identified in case reports of systemic arterial gas embolism occurring during bronchoscopic biopsy procedures

\begin{tabular}{|c|c|}
\hline & All patients $(n=29)$ \\
\hline \multicolumn{2}{|l|}{ Clinical manifestations } \\
\hline Cerebral & $28(97 \%)$ \\
\hline Intraprocedural unresponsiveness & $13(45 \%)$ \\
\hline Post-procedural AMS without focal deficit & $8(28 \%)$ \\
\hline Seizure & $14(48 \%)$ \\
\hline Focal deficits & $15(52 \%)$ \\
\hline Left sided & $13(45 \%)$ \\
\hline Right sided & $2(7 \%)$ \\
\hline Cardiac & $8(28 \%)$ \\
\hline Cardiac arrest & $5(17 \%)$ \\
\hline Bradycardia & $8(28 \%)$ \\
\hline ST elevations & $5(17 \%)$ \\
\hline Complete heart block & $3(10 \%)$ \\
\hline Chest pain & $2(7 \%)$ \\
\hline \multicolumn{2}{|l|}{ Diagnosis } \\
\hline \multicolumn{2}{|l|}{ Likely embolic location of gas* } \\
\hline Cerebral only & $20(69 \%)$ \\
\hline Coronary only & $1(3 \%)$ \\
\hline Cerebral and coronary & $8(30 \%)$ \\
\hline Confirmation of gas embolism & $23(79 \%)$ \\
\hline CT head & $19(66 \%)$ \\
\hline Other $^{\Delta}$ & $4(14 \%)$ \\
\hline \multicolumn{2}{|l|}{ Treatment } \\
\hline Supplemental oxygen therapy & $27(93 \%)$ \\
\hline Normobaric & $16(55 \%)$ \\
\hline Hyperbaric & $11(38 \%)$ \\
\hline Manual gas aspiration & $2(7 \%)$ \\
\hline Antiepileptic therapy & $9(31 \%)$ \\
\hline tPA administration & $1(3 \%)$ \\
\hline \multicolumn{2}{|l|}{ Clinical outcome } \\
\hline Full recovery & $14(48 \%)$ \\
\hline Residual deficits & $7(24 \%)$ \\
\hline Death & $8(28 \%)$ \\
\hline
\end{tabular}

AMS, altered mental status; CT, computed tomography; tPA, tissue plasminogen activator. ${ }^{*}$, based on clinical and radiographic features; ${ }^{\triangle}$, includes coronary angiogram, chest $\mathrm{x}$-ray, dilated retinal exam, and autopsy findings. Categorical measures reported as number (percentage). 
improvement or lack of institutional hyperbaric oxygen therapy were cited as reasons for the implementation of normobaric oxygen therapy alone. Other treatments included antiepileptics (in cases where seizures occurred), 2 reports of attempted direct gas aspiration, and 1 report of thrombolytic administration (followed by severe hemoptysis). The mortality rate in this series was $28 \%$, with $24 \%$ having residual deficits at time of case report; almost half of patients (48\%) demonstrated full recovery. Mortality was higher in patients who received hyperbaric therapy (36\%) compared to normobaric therapy $(20 \%)$, but rates of full recovery in the two groups were equivalent $(45 \%$ and $47 \%$ respectively).

\section{Discussion}

\section{Pathophysiology and epidemiology}

Minute gas bubbles can cause occlusion of functional end arterioles in any arterial bed, leading to ischemia (33); however, clinically-significant occlusions are predominantly seen in the cerebral and coronary circulations. Though gas embolism is classically associated with depressurization injury, reported cases are much more commonly due to traumatic or iatrogenic introduction of gas. Routes of entry to the arterial circulation include direct systemic arterial or pulmonary venous entrainment of gas, or systemic venous gas passing via a right-to-left shunt. SAGE from the pulmonary venous circulation can occur after penetrating thoracic trauma or iatrogenically during cardiovascular surgery, percutaneous lung biopsy, or bronchoscopic lung biopsy.

The incidence of SAGE during flexible bronchoscopy has been reported as less than $0.02 \%(18,30)$. In the largest survey of bronchoscopic complications, Asano et al. describe only 2 reported cases of systemic gas embolism out of 103,978 flexible bronchoscopies; however, both were fatal (1). This is in contradistinction to the rates of SAGE during percutaneous lung biopsy, which are reported to be between $0.06 \%$ (34) and $0.9 \%$ (35) in the latter study, 16 of 19 instances of SAGE were asymptomatic and only identified during routine head and chest CT after biopsy. A retrospective review of 559 percutaneous lung biopsy cases at one institution revealed a radiographic incidence of $4.8 \%$, despite a clinical incidence of only $0.17 \%$ (36). These findings suggest that asymptomatic SAGE may be much more common after bronchoscopic biopsy than is currently known, and may contribute to the otherwiseidiopathic acute cardiovascular complications associated with bronchoscopy-cited as $0.068 \%$ by Asano et al.

Awareness of this complication during bronchoscopic biopsy appears low, though it may be growing, with twentytwo of the twenty-nine cases in our series published within the last ten years. There appears to be greater awareness regarding the risk of SAGE during ablative airway procedures (2). Multiple case reports did not acutely consider SAGE as a possible etiology despite acute cardiac and neurologic complications consistent with this diagnosis (31,32). Amongst other published case series, fewer than half of the known case reports published at the time were included (19), and few discussed the possibility of coronary emboli despite acute cardiogenic manifestations. The complication may also be underreported even when recognized (37) given the severe consequences to the patient and possible professional and legal ramifications to proceduralists.

Risk factors for SAGE during bronchoscopic biopsy procedures likely correlate with the two conditions required for entrance of gas into the pulmonary venous circulation: a direct connection between an airspace and pulmonary vein, and a pressure gradient sufficient to drive gas from the airspace into the venous circulation. Vessel injury may create a transient broncho- or alveolo-venous fistula leading to risk of SAGE. Indeed moderate-tosevere bleeding is commonly reported in cases of SAGE following bronchoscopic biopsy, though direct confirmation of damaged pulmonary venous structures on pathology is rare $(6,38)$. Positive pressure ventilation may create a pressure gradient for airflow into the pulmonary venous circulation and appears to be a risk factor for SAGE in the case literature of percutaneous lung biopsy $(35,39)$ and penetrating thoracic trauma (40). Our case describes an instance of SAGE occurring during a recruitment manuever and an episode of SAGE included in our series occurred directly after oxygen insufflation into a collapsed airway, both demonstrating the potentially-deleterious effect of increased positive airway pressure (9). Most reported cases of SAGE during bronchoscopic biopsy occurred in spontaneously breathing patients; however transient rises in airway pressures, such as with coughing [which can create local pressure in excess of $\left.180 \mathrm{cmH}_{2} \mathrm{O}(41)\right]$, could generate alveolar-venous airflow, especially with wedging of the bronchoscope or if obstructive lung disease causes gas trapping. Coughing after biopsy and obstructive lung disease are indeed reported risk factors for SAGE after percutaneous lung biopsy (35).

In this series, SAGE occurred after multiple bronchoscopic 
Table 3 Clinical manifestations suggesting the possibility of SAGE during/after bronchoscopic biopsy procedures

\section{During procedure \\ Hypotension or cardiovascular collapse \\ Bradycardia, heart block, or ST elevations}

Seizure or abnormal extremity movements

Acute unresponsiveness or comatose state

During recovery

Abnormal movements, suggesting seizure

New focal neurologic deficits, especially hemiparesis

Delayed awakening from anesthesia

Non-specific agitation, confusion, or lethargy

SAGE, systemic arterial gas embolism.

biopsy techniques, with numerically more cases after forceps biopsy than needle aspiration. One can surmise that larger or more numerous biopsies are more likely to lead to SAGE. Indeed, an incidence of SAGE was noted in a recent review of safety and diagnostic outcomes after transbronchial cryobiopsy, with a resulting incidence rate of $0.3 \%$, far greater than published reports from other transbronchial biopsy methods (42). Other possible risk factors for SAGE include the location of biopsy (reported more commonly in the lower lobes, especially on the right (15) and the presence of a cavitary mass (19); however in our series, SAGE occurred after biopsies in all lobes, and only a small minority were associated with cavitary lesions. The fact that a greater number of cases occurred under moderate sedation than with topicalization alone likely relates to the prevalence of these techniques during routine bronchoscopy, rather than suggesting variable risks of SAGE. Prospective studies would be needed to better characterize the potential risk of SAGE from variability in procedural techniques.

\section{Clinical manifestations}

SAGE after bronchoscopic biopsy generally appears to follow two distinct temporal patterns (Table 3). The first and more common is an immediate neurologic or cardiovascular emergency, often within minutes of biopsy. Acute neurologic manifestations include focal neurologic deficits (especially hemiparesis), seizures, obtundation, and unresponsiveness. Acute cardiovascular manifestations are rarer and include arrhythmias [most commonly bradycardia/sinus arrest and atrioventricular (AV) blocks, although ventricular tachyarrhythmias are also reported], myocardial ischemia, and cardiovascular collapse. Based on our literature review, cardiovascular manifestations are almost always associated with concurrent cerebral gas embolism $(17,43)$. The second pattern identified is a delayed neurologic presentation with altered mental status or agitation noted after the cessation of sedation. Unfortunately, few other signs exist to suggest the possibility of gas embolism, though marbling of the skin has been noted-especially in anterior or non-dependent regions of the body (44).

The most common focal neurologic manifestation is hemiparesis, correlating with involvement of the contralateral middle cerebral artery (MCA). Seizures are acute, often generalized tonic-clonic, and much more frequent than the incidence of $2-4 \%$ seen after thromboembolic ischemia (45). Unresponsiveness or obtundation may be due to frontal lobe involvementoften bilateral and mediated by anterior cerebral artery (ACA) embolization-or due to a post-ictal state. The tendency for MCA and ACA involvement is likely related to supine positioning during bronchoscopy, with gas emboli commonly tracking anti-gravitationally to non-dependent vascular territories. The tendency for left-sided symptoms is notable in our case series, perhaps owing to the more anterior position of the brachiocephalic artery-and, by extension, the right internal carotid artery—along the aortic arch; indeed, this pattern was notable in our case which was performed in the supine position. Although most cases in this series did not report patient positioning, supine positioning can likely be assumed for most bronchoscopic procedures. In the two reports of procedures performed in the left lateral decubitus position, both clinical and radiographic features were also consistent with right-sided infarcts, as demonstrated by dense left hemiplegia and gas emboli visualized within right MCA and PCA territories $(16,19)$. Posterior cerebral circulation involvement is associated with prone positioning during percutaneous lung biopsy (46) and thus appears to be rare during bronchoscopy; only one case reported isolated cerebellar and occipital embolism, associated with post-procedure ataxia and dysarthria (14), and patient positioning was not recorded. Another case noted isolated posterior emboli in the right occipital lobe on initial imaging; however followup MRI revealed diffuse right-sided involvement in the frontal and parietal lobes as well, suggesting a more diffuse cerebral embolic process (28). 

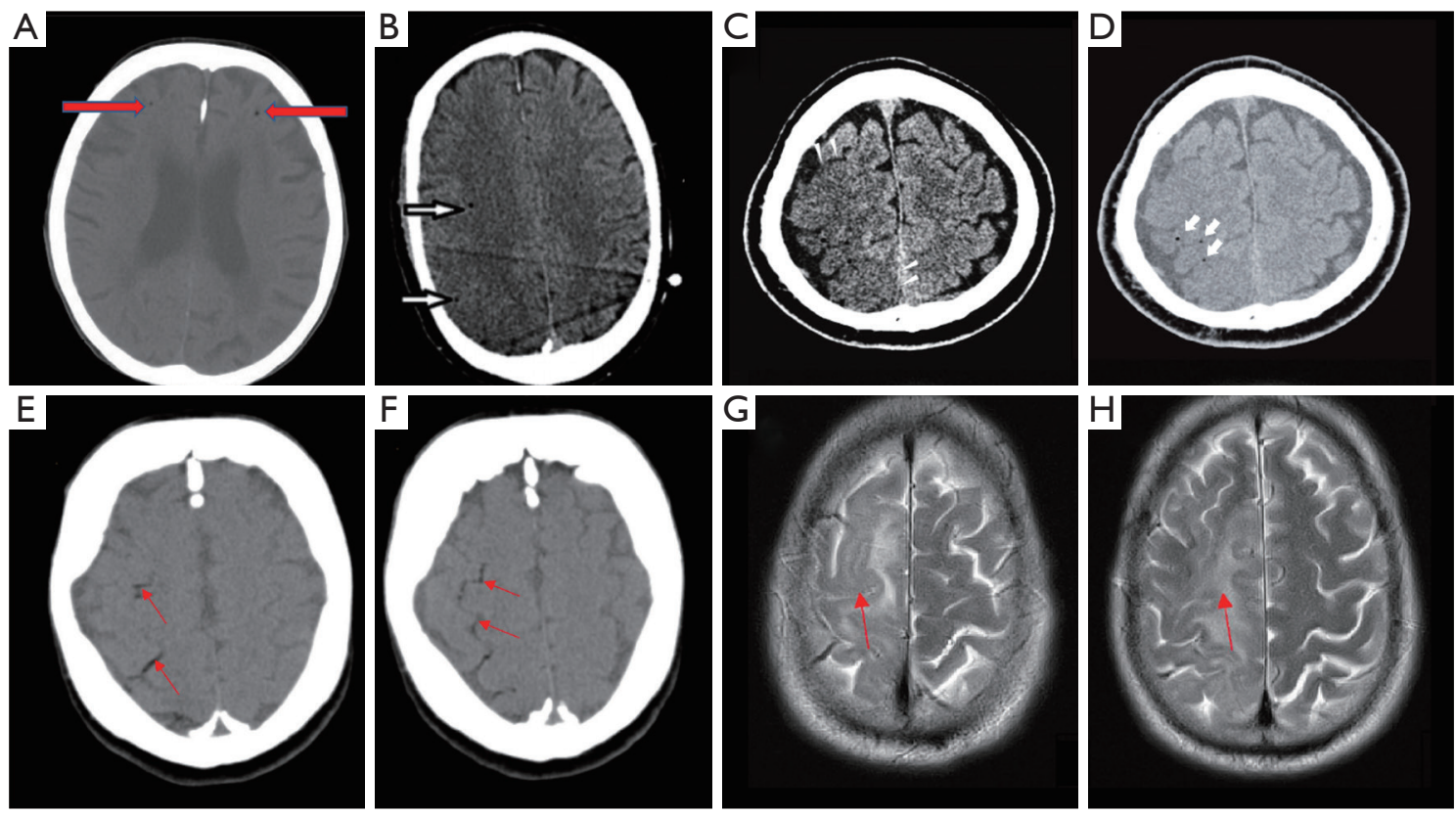

Figure 5 Confirmation of SAGE by neurologic imaging after bronchoscopic biopsy procedures. Gas emboli visualized on CT are commonly located near the grey and white matter junction, in the anterior cerebral artery [red arrows in A, courtesy Evison et al. (15)] and middle cerebral artery [white arrows in B, courtesy Fogelfeld et al. (22)] territories. Gas emboli not visible on brain windows (C, white arrows denoting obscuration of the corticomedullary junction) may become evident by utilizing mediastinal windows [D, white arrows, courtesy Maemura et al. (19)]. CT may also demonstrate gas collections in the extra-axial space (red arrows, E,F), in this case concomitant with changes in MRI within the right middle cerebral artery territory [red arrows, $(\mathrm{G}, \mathrm{H})$ courtesy Almas et al. (29)]. All images reprinted with permission. MRI, magnetic resonance imaging; SAGE, systemic arterial gas embolism.

Gas emboli in the coronary circulation also appear to follow anti-gravitational patterns, again depending on patient positioning. The literature on percutaneous lung biopsy suggests that the supine and left lateral positions are more commonly associated with right coronary artery involvement, as its ostium is more non-dependent in these positions (38). Although acute bradycardia and AV nodal blockade could potentially be due to a strong vagal response after cerebral embolization, bradyarrhythmias are far less common than tachyarrhythmias in acute stroke $(18,47,48)$, and in an animal model, cerebral gas emboli induced in cats led to acute hypertension and tachyarrhythmias, whereas bradycardia and heart block were not reported (49). Furthermore, ST segment changes localizing to the inferior leads, especially in the context of chest pain, are most consistent with a focal occlusion of the right coronary artery. Our case demonstrated inferior ST segment elevations, bradycardia and AV nodal blockade, and similar findings had a high frequency in our review of published cases with cardiac involvement-all consistent with preferential right coronary artery involvement in the supine position. Acute right ventricular failure from proximal right coronary artery (RCA) occlusion could also explain the cases of complete cardiovascular collapse noted in this series $(6,18,19,22,31)$, compounding neurologic injury from direct cerebral gas embolism (with which it is often concurrent).

\section{Diagnostic imaging}

The diagnosis of SAGE can be confirmed by visualization of gas emboli in systemic organs, either directly (in the case of gas emboli visible in retinal arteries via ophthalmoscopy, or from aspiration of gas during angiography of the associated vessels), or, far more commonly, radiographically. Representative neurologic imaging in cases of confirmed SAGE is shown in Figure 5. CT of the head is the most common modality for visualizing gas emboli, which appear as small, well-defined regions of low density. Utilizing lung windowing rather than bone or brain windowing can assist in identifying such areas within the cortical matter (19). 
Similar to other arterial embolic phenomena, arterial gas emboli are most commonly visualized at the gray-white matter junction, although they can collect in serpiginous patterns in the extra-axial space; by comparison, venous gas emboli traveling in a retrograde fashion from a site of systemic venous injury will often collect along the cortical sulci (50). Brain MRI with diffusion-weighted imaging will demonstrate gyriform infarcts or vasogenic edema, often associated with the locations of gas emboli seen on CT (51). As discussed previously, the distal watershed zones of the MCA and ACA appear to be at highest risk; these areas correspond to low flow areas in the end-arterial cortical layer where small gas bubbles can be trapped and prevent reperfusion (52).

The fact that $21 \%$ of included case reports, including our own case, could not directly visualize gas emboli in the cerebral circulation is consistent with the literature on radiographic imaging of systemic gas emboli. The volume of embolized gas necessary to cause fatal injury in either the cerebral or coronary circulations is thought to be on the order of 0.5 to $2 \mathrm{~cm}^{3}$ (53); as seen in dogs who received this volume of gas injected intraarterially, with a half-life of gas absorption of 8 minutes (54). Furthermore, it is known that gas emboli as large as $1 \mathrm{~cm}^{3}$ may not be visualized via any imaging modality once diffusely distributed (29). In humans diagnosed with decompression sickness, CT and MRI imaging is often unable to identify gas emboli $(55,56)$, and in a consecutive series of 16 patients referred for hyperbaric treatment at one institution after presumed SAGE who underwent imaging, only 2 demonstrated abnormalities on CT or MRI despite ongoing symptoms (57). One report of cerebral gas embolism after percutaneous lung biopsy was unable to demonstrate intraparenchymal gas even on CT 30 minutes after the clinical event (58), and another showed complete resolution of previous "massive" gas emboli only two hours after the event (27). Brain MRI often reveals widespread areas of restricted diffusion which do not correlate with affected areas visualized on CT (59); these infarcts may be related to the after-effects of vasospasm and vascular inflammation, which remain despite rapid gas resorption.

Coronary gas emboli can occasionally be confirmed fluoroscopically during coronary catheterization, though the rarity of rapid coronary angiography precludes an estimate of its sensitivity for detecting gas emboli. In contradistinction to thromboemboli which lead to angiographic vessel cutoff, gas emboli are often associated with vaguely-defined areas of low flow (60). Direct gas aspiration has been utilized to treat acute coronary gas embolism (17), and angiography can rule out significant thromboembolic disease as an alternative etiology for myocardial ischemia or infarction.

Overall, imaging confirmation of gas emboli likely depends on the volume of gas and the timing of imaging, as well as on the degree of clinical suspicion on the part of the treating providers. Given the imperfect sensitivity of standard imaging techniques, it is important for the bronchoscopist to be aware of the limitations of imaging for the diagnosis of SAGE, and maintain a high index of suspicion in the presence of an appropriate risk factor and consistent clinical syndrome.

\section{Treatment, prognosis, and prevention}

The mainstays of acute management in SAGE after bronchoscopic biopsy are rapid recognition, termination of the biopsy procedure, acute cardiovascular resuscitation as necessary, and initiation of oxygen therapy. If gas remains in the left heart or large vessels, right lateral decubitus or supine positioning may theoretically help to avoid further cerebral embolization by trapping gas in the left ventricular apex, in contradistinction to left lateral Trendelenburg positioning (Durant's maneuver) for systemic venous gas embolism (61). If utilizing intra-procedural CT, a rapid sequence of head and chest imaging can be obtained which may confirm the diagnosis of SAGE.

In all cases of SAGE, 100\% supplemental oxygen should be immediately administered. If intubated, airway pressures should be limited in order to prevent a persistent airwayto-vein pressure differential. The principles of oxygen use are twofold: first, an increase in the oxygen tension facilitates absorption of nitrogen from gas bubbles, and second, high oxygen tension maximizes perfusion and oxygenation in obstructed vascular beds distal to the gas embolus. Hyperbaric oxygen therapy (HBOT) additionally reduces the volume of in-situ gas bubbles in vasculature via Boyle's Law (62). Although there are no prospective randomized controlled studies proving the efficacy of HBOT, the strong physiologic mechanisms and supportive data from human cohorts and animal studies (63) suggest a clinically significant effect with a likely mortality benefit, which may be effective despite delays in initiation (64). In divers experiencing gas embolism, delays in HBOT greater than 6 hours are not associated with worse outcomes (57). One case in this review demonstrated dramatic improvement in neurologic function within 90 minutes of 
completing an 8-hour HBOT treatment session, despite remaining comatose during the 48-hour delay between the procedure and HBOT therapy. Although in this review we reported outcomes stratified by the use of hyperbaric or normobaric oxygen, these are likely strongly influenced by the high likelihood of significant bias related to clinical severity (which likely associates with consideration for the use of hyperbaric oxygen) and should not dissuade from the use of hyperbaric therapy. Therefore, after the clinical diagnosis of SAGE, institution of HBOT should be considered, including transfer to a HBOT-capable facility. In this review, most patients did not receive HBOT either because their diagnosis was unrecognized, their condition improved rapidly with normobaric oxygen therapy, or they were deemed unsuitable for transport due to their clinical condition. Lastly, a multidisciplinary discussion with emergency neurologic and cardiac consultants about the possibility of SAGE may help to avoid aggressive empiric therapy for thromboembolic disease-particularly systemic fibrinolytic therapy which can lead to life-threatening hemoptysis after biopsy (29).

Mortality due to SAGE is unfortunately high; the mortality rate of $28 \%$ observed in this review is similar to that seen among patients with SAGE after percutaneous lung biopsy (38). However this review suggests that, as seen in our case, most neurologic deficits among survivors resolve completely within days of the event, and the majority of survivors have no significant long-term neurologic deficits, with a minority suffering from prolonged sequelae $(7,15,19)$. If resuscitated successfully, most patients with cardiovascular involvement demonstrate no long-term cardiac disease, though isolated wall motion abnormalities at discharge were noted in one case (17). This is consistent with animal models demonstrating rapid return of systolic function after acute coronary gas embolism (65). Isolated gas embolism during coronary angiography is associated with a much lower mortality rate of approximately $3 \%$ (66), although this may be related to rapid aspiration and smaller volumes of arterial gas than likely occur with bronchoscopic procedures.

Unfortunately, prevention of SAGE during bronchoscopy may be difficult; there is likely some degree of unmitigable risk from violating the lung parenchyma via any approach. Some preventative strategies during percutaneous lung biopsy may be transferrable to bronchoscopy, including use of the small-diameter needles, performing biopsies only during breath holds, and occluding the hollow needle after stylet removal $(67,68)$. Increased sedation and topical analgesia for patients who are coughing/straining during bronchoscopy could theoretically limit airway pressure swings, and positive pressure ventilation can be limited or avoided (39). Early identification of asymptomatic gas embolism may be possible during bronchoscopic procedures, via modalities employed in cardiac or vascular procedures including transcranial doppler monitoring with associated electroencephalography (69), transesophageal echocardiography of the left ventricle or right coronary artery to visualize systemic arterial gas emboli $(70,71)$, and near-infrared spectroscopy (NIRS) to evaluate cerebral oxygen uptake $(27,72)$. Given the low incidence of SAGE, it is unlikely that these will be routinely employed during bronchoscopy.

\section{Limitations}

The implications of this review are limited by the retrospective nature of a literature review; it is reasonable to assume that cases of SAGE with dramatic presentations are more likely to be published, leading to publication bias and a potential for higher rates of morbidity and mortality than would be seen prospectively. However, the rates of SAGE appear low enough that prospective study during routine bronchoscopy may not be feasible. It also remains possible that severe cases are under-reported due to under-diagnosis or concerns with professional or legal ramifications. Although we strove to systematically include all available studies in this literature review, it is possible that there exist other case reports of SAGE not available at least in abstract form at the time of literature review.

\section{Conclusions}

Although rare, SAGE is nevertheless an underrecognized and potentially catastrophic adverse event of bronchoscopic biopsy procedures. The diagnosis is primarily clinical in the setting of peri-procedural neurologic manifestations (whether acute or delayed), which may co-occur with acute cardiac presentations. In such cases, it is imperative that the bronchoscopist maintain a high index of suspicion for SAGE, even in the absence of confirmatory imaging, and consider prompt initiation of treatment, including high-concentration hyperbaric oxygen, to prevent morbidity and mortality.

\section{Acknowledgments}

Funding: None. 


\section{Footnote}

Reporting Checklist: The authors have completed the PRISMA reporting checklist. Available at https://dx.doi. org/10.21037/jtd-21-717

Peer Review File: Available at https://dx.doi.org/10.21037/ jtd-21-717

Conflicts of Interest: All authors have completed the ICMJE uniform disclosure form (available at https://dx.doi. org/10.21037/jtd-21-717). The authors have no conflicts of interest to declare.

Ethical Statement: The authors are accountable for all aspects of the work in ensuring that questions related to the accuracy or integrity of any part of the work are appropriately investigated and resolved.

Open Access Statement: This is an Open Access article distributed in accordance with the Creative Commons Attribution-NonCommercial-NoDerivs 4.0 International License (CC BY-NC-ND 4.0), which permits the noncommercial replication and distribution of the article with the strict proviso that no changes or edits are made and the original work is properly cited (including links to both the formal publication through the relevant DOI and the license). See: https://creativecommons.org/licenses/by-nc-nd/4.0/.

\section{References}

1. Asano F, Aoe M, Ohsaki Y, et al. Deaths and complications associated with respiratory endoscopy: a survey by the Japan Society for Respiratory Endoscopy in 2010. Respirology 2012;17:478-85.

2. Reddy C, Majid A, Michaud G, et al. Gas embolism following bronchoscopic argon plasma coagulation: a case series. Chest 2008;134:1066-9.

3. Kanchustambham V, Saladi S, Mehta K, et al. Vascular Air Embolism During Bronchoscopy Procedures- Incidence, Pathophysiology, Diagnosis, Management and Outcomes. Cureus 2017;9:e1087.

4. Moher D, Shamseer L, Clarke M, et al. Preferred reporting items for systematic review and meta-analysis protocols (PRISMA-P) 2015 statement. Syst Rev 2015;4:1.

5. Erickson AD, Irwin RS, Teplitz C, et al. Cerebral air embolism complicating transbronchoscopic lung biopsy. Ann Intern Med 1979;90:937-8.

6. Strange C, Heffner JE, Collins BS, et al. Pulmonary hemorrhage and air embolism complicating transbronchial biopsy in pulmonary amyloidosis. Chest 1987;92:367-9.

7. Ayers ML, Manning H. Cerebral air embolism complicating transbronchial needle aspiration. J Bronchology Interv Pulmonol 1999;6:137.

8. Shetty PG, Fatterpekar GM, Manohar S, et al. Fatal cerebral air embolism as a complication of transbronchoscopic lung biopsy: a case report. Australas Radiol 2001;45:215-7.

9. Wherrett CG, Mehran RJ, Beaulieu MA. Cerebral arterial gas embolism following diagnostic bronchoscopy: delayed treatment with hyperbaric oxygen. Can J Anaesth 2002;49:96-9.

10. Dhillon SS, Agrawal A, Gorin AB. Cerebral arterial air embolism after transbronchial lung biopsy: a case report and review of literature. J Bronchology Interv Pulmonol 2004;11:125-7.

11. Maricich SM, Rauch RA, Foreman PJ. Cerebral air embolism during transbronchial biopsy. Neurology 2004;62:1438.

12. Delannoy B, Bayle F, Arpin D, et al. Cerebral gas arterial embolism complicating bronchial biopsies with a flexible bronchoscope: a case report. Intensive Care Med 2011;37:555-6.

13. Perinel Ragey S, Garnier P, Vergnon JM. Complete resolution of cerebral air embolism secondary to a transbronchial needle aspiration. Respiration 2013;86:504-7.

14. Dong H, Grimes B, Oh S, et al. Airways and Airheads: A Case of Bronchoscopy-Induced Cerebral Arterial Gas Embolism. Chest 2014;146:791A.

15. Evison M, Crosbie PA, Bright-Thomas R, et al. Cerebral air embolism following transbronchial lung biopsy during flexible bronchoscopy. Respir Med Case Rep 2014;12:39-40.

16. Goto H, Nakao M, Ohyanagi F. A case of cerebral air embolism following bronchoscopy. The Journal of the Japan Society for Respiratory Endoscopy 2014;36:649-55.

17. Sumi T, Tanaka A, Ishii H, et al. Coronary air embolism following transbronchial lung biopsy. Cardiovasc Interv Ther 2019;34:64-6.

18. Tsuji T, Sonobe S, Koba T, et al. Systemic Air Embolism Following Diagnostic Bronchoscopy. Intern Med 2017;56:819-21.

19. Maemura K, Kage H, Isago H, et al. Cerebral Arterial Air Embolism after Diagnostic Flexible Fiberoptic Bronchoscopy: A Case Report and Review of the Literature. Case Rep Pulmonol 2018;2018:7108215.

20. Mauricio S, Rebêlo MI. Unresponsive patient after flexible 
bronchoscopy - a fatal case of cerebral air embolism. 2018. Presented at: Euroanesthesia, June 1-3, 2018, Copenhagen, Denmark.

21. Galante O, Fuchs L, Almog Y, et al. Cerebral air emboli complicating trans-bronchial needle aspiration using endobronchial ultrasound. Intensive Care Med 2018;44:98-9.

22. Fogelfeld K, Rana RK, Soo Hoo GW. Cerebral Artery Gas Embolism Following Navigational Bronchoscopy. J Intensive Care Med 2018;33:536-40.

23. Toyota H, Kobayashi K, Matsukura S, et al. A Case of Cerebral Air Embolism During Diagnostic Flexible Bronchoscopy. J Bronchology Interv Pulmonol 2019;26:e58-60.

24. Agossou M, Holtea-Souty D, Fore M, et al. Cerebral air embolism: A rare complication of flexible fiberoptic bronchoscopy. Rev Mal Respir 2020;37:662-5.

25. Brevig R, Hamer L, Baquis G, et al. Delayed Cerebral Air Embolization after Transbronchoscopic Lung Biopsy. Neurology 1998; 50(4).

26. Seeburger J, Borger MA, Merk DR, et al. Massive cerebral air embolism after bronchoscopy and central line manipulation. Asian Cardiovasc Thorac Ann 2009;17:67-9.

27. Matsumoto H, Umakoshi K, Takeba J, et al. Continuous Monitoring of Cerebral Near-Infrared Spectroscope (NIRS) after Cerebral Air Embolism. Emergency Medicine: Open Access 2015;5:1000252.

28. Van Den Plas K, Van den Bergh V, Van Grimberge F, et al. Cerebral Arterial Air Embolism After Endobronchial Ultrasound-guided Transbronchial Needle Aspiration. J Bronchology Interv Pulmonol 2020;27:e62-4.

29. Almas ET, Casserly B. Air embolism following bronchoscopy with fine needle aspiration: An unexpected complication. Respir Med Case Rep 2018;25:228-32.

30. Azzola A, von Garnier C, Chhajed PN, et al. Fatal cerebral air embolism following uneventful flexible bronchoscopy. Respiration 2010;80:569-72.

31. Kumar S, Nath A, Singh S, et al. An unusual complication during bronchoscopy: hypotension, global ST segment elevation, and acute severe left ventricular systolic dysfunction. Respir Care 2013;58:e111-5.

32. Mohan A, Kumar A, Dhillon SS. C41 Case reports in interventional pulmonary: Seizures After A Bronchoscopy: A Cautionary Tale With Sarcoidosis. AJRCCM 2014;189:A4461.

33. Muth CM, Shank ES. Gas embolism. N Engl J Med 2000;342:476-82.

34. Tomiyama N, Yasuhara Y, Nakajima Y, et al. CTguided needle biopsy of lung lesions: a survey of severe complication based on 9783 biopsies in Japan. Eur J Radiol 2006;59:60-4.

35. Liu SH, Fu Q, Yu HL, et al. A retrospective analysis of the risk factors associated with systemic air embolism following percutaneous lung biopsy. Exp Ther Med 2020;19:347-52 .

36. Monnin-Bares V, Chassagnon G, Vernhet-Kovacsik H, et al. Systemic air embolism depicted on systematic whole thoracic CT acquisition after percutaneous lung biopsy: Incidence and risk factors. Eur J Radiol 2019;117:26-32.

37. Haber AD. The Law of Unintended Consequences. Ann Intern Med 2016;165:824-5.

38. Ibukuro K, Tanaka R, Takeguchi T, et al. Air embolism and needle track implantation complicating CT-guided percutaneous thoracic biopsy: single-institution experience. AJR Am J Roentgenol 2009;193:W430-6.

39. Kazimirko DN, Beam WB, Saleh K, et al. Beware of positive pressure: coronary artery air embolism following percutaneous lung biopsy. Radiol Case Rep 2016;11:344-7.

40. Thomas AN, Roe BB. Air embolism following penetrating lung injuries. J Thorac Cardiovasc Surg 1973;66:533-40.

41. Agostoni E, Rahn H. Abdominal and thoracic pressures at different lung volumes. J Appl Physiol 1960;15:1087-92.

42. Matsumoto Y, Nakai T, Tanaka M, et al. Diagnostic Outcomes and Safety of Cryobiopsy Added to Conventional Sampling Methods: An Observational Study. Chest 2021. [Epub ahead of print].

43. Hsi DH, Thompson TN, Fruchter A, et al. Simultaneous coronary and cerebral air embolism after CT-guided core needle biopsy of the lung. Tex Heart Inst J 2008;35:472-4.

44. Durant TM, Oppenheimer MJ. Arterial air embolism. Am Heart J 1949;38:481-500.

45. Camilo O, Goldstein LB. Seizures and epilepsy after ischemic stroke. Stroke 2004;35:1769-75.

46. Droghetti L, Giganti M, Memmo A, et al. Air embolism: diagnosis with single-photon emission tomography and successful hyperbaric oxygen therapy. Br J Anaesth 2002;89:775-8.

47. Mikolich JR, Jacobs WC, Fletcher GF. Cardiac arrhythmias in patients with acute cerebrovascular accidents. JAMA 1981;246:1314-7.

48. Kallmünzer B, Breuer L, Kahl N, et al. Serious cardiac arrhythmias after stroke: incidence, time course, and predictors--a systematic, prospective analysis. Stroke 2012;43:2892-7.

49. Evans DE, Kobrine AI, Weathersby PK, et al. Cardiovascular effects of cerebral air embolism. Stroke 1981;12:338-44A.

50. Brouns R, De Surgeloose D, Neetens I, et al. Fatal venous 
cerebral air embolism secondary to a disconnected central venous catheter. Cerebrovasc Dis 2006;21:212-4.

51. Jeon SB, Kim JS, Lee DK, et al. Clinicoradiological characteristics of cerebral air embolism. Cerebrovasc Dis 2007;23:459-62.

52. Caplan LR, Hennerici M. Impaired clearance of emboli (washout) is an important link between hypoperfusion, embolism, and ischemic stroke. Arch Neurol 1998;55:1475-82.

53. Bou-Assaly W, Pernicano P, Hoeffner E. Systemic air embolism after transthoracic lung biopsy: A case report and review of literature. World J Radiol 2010;2:193-6.

54. Annane D, Troché G, Delisle F, et al. Kinetics of elimination and acute consequences of cerebral air embolism. J Neuroimaging 1995;5:183-9.

55. Hodgson M, Beran RG, Shirtley G. The role of computed tomography in the assessment of neurologic sequelae of decompression sickness. Arch Neurol 1988;45:1033-5.

56. Levin HS, Goldstein FC, Norcross K, et al. Neurobehavioral and magnetic resonance imaging findings in two cases of decompression sickness. Aviat Space Environ Med 1989;60:1204-10.

57. Trytko BE, Bennett MH. Arterial gas embolism: a review of cases at Prince of Wales Hospital, Sydney, 1996 to 2006. Anaesth Intensive Care 2008;36:60-4.

58. Suzuki K, Ueda M, Muraga K, et al. An unusual cerebral air embolism developing within the posterior circulation territory after a needle lung biopsy. Intern Med 2013;52:115-7.

59. Kaichi Y, Kakeda S, Korogi Y, et al. Changes over Time in Intracranial Air in Patients with Cerebral Air Embolism: Radiological Study in Two Cases. Case Rep Neurol Med 2015;2015:491017.

60. Kramer M, Parekh M, Modi RY, et al. Review of Thoracic Causes of Systemic Arterial Air Embolism on Computed Tomography. J Thorac Imaging 2020;35:W68-74.

61. Balsara ZN, Burks DD. Hyperbaric oxygen therapy for arterial air embolism. AJR Am J Roentgenol 2007;188:W98; author reply W99.

62. van Hulst RA, Klein J, Lachmann B. Gas embolism:

Cite this article as: Swenson KE, Shaller BD, Duong K, Bedi H. Systemic arterial gas embolism (SAGE) as a complication of bronchoscopic lung biopsy: a case report and systematic literature review. J Thorac Dis 2021;13(11):6439-6452. doi: $10.21037 /$ jtd-21-717 pathophysiology and treatment. Clin Physiol Funct Imaging 2003;23:237-46.

63. Layon AJ. Hyperbaric oxygen treatment for cerebral air embolism--where are the data? Mayo Clin Proc 1991;66:641-6.

64. Baskin SE, Wozniak RF. Hyperbaric oxygenation in the treatment of hemodialysis-associated air embolism. N Engl J Med 1975;293:184-5.

65. Van Blankenstein JH, Slager CJ, Schuurbiers JC, et al. Heart function after injection of small air bubbles in coronary artery of pigs. J Appl Physiol (1985) 1993;75:1201-7.

66. Kariyanna PT, Jayarangaiah A, Jayarangaiah A, et al. Coronary Air Embolism During Coronary Angiography : A Systematic Review. Scifed J Cardiol 2018.

67. Rott G, Boecker F. Influenceable and Avoidable Risk Factors for Systemic Air Embolism due to Percutaneous CT-Guided Lung Biopsy: Patient Positioning and Coaxial Biopsy Technique-Case Report, Systematic Literature Review, and a Technical Note. Radiol Res Pract 2014;2014:349062.

68. Glodny B, Schönherr E, Freund MC, et al. Measures to Prevent Air Embolism in Transthoracic Biopsy of the Lung. AJR Am J Roentgenol 2017;208:W184-91.

69. Yeh T Jr, Austin EH 3rd, Sehic A, et al. Rapid recognition and treatment of cerebral air embolism: the role of neuromonitoring. J Thorac Cardiovasc Surg 2003;126:589-91.

70. Saada M, Goarin JP, Riou B, et al. Systemic gas embolism complicating pulmonary contusion. Diagnosis and management using transesophageal echocardiography. Am J Respir Crit Care Med 1995;152:812-5.

71. Gourav KP, Bhat I, Damodaran S, et al. Detection of right coronary artery air embolism by transesophageal echocardiography. Journal of Perioperative Echocardiography 2018;6:29-32.

72. Casati A, Spreafico E, Putzu M, et al. New technology for noninvasive brain monitoring: continuous cerebral oximetry. Minerva Anestesiol 2006;72:605-25. 